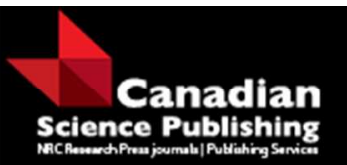

Canadian Journal of Forest Research

Revue canadienne de recherche forestière

\title{
An improved estimator for the sampling error of local competition variables
}

\begin{tabular}{|r|l|}
\hline Journal: & Canadian Journal of Forest Research \\
\hline Manuscript ID & cjfr-2015-0162.R1 \\
\hline Manuscript Type: & Note \\
\hline Date Submitted by the Author: & 10 -Aug-2015 \\
\hline Complete List of Authors: & $\begin{array}{l}\text { Antón-Fernández, Clara; Norwegian Institute of Bioeconomy Research } \\
\text { Froese, Robert; Michigan Technological University }\end{array}$ \\
\hline Keyword: & $\begin{array}{l}\text { measurement error, PBA, point basal area, structural based prediction, } \\
\text { competition index }\end{array}$ \\
\hline \multicolumn{2}{|l}{} \\
\hline
\end{tabular}

SCHOLARONE ${ }^{\text {M }}$

Manuscripts 


\title{
An improved estimator for the sampling error of
} local competition variables

\author{
Clara Antón-Fernández and Robert E. Froese
}

Clara Antón-Fernández. ${ }^{1}$ Norwegian Institute of Bioeconomy Research, Postboks 115, 1431 Ås, Norway. e-mail: caf@nibio.no

Robert E. Froese. Michigan Technological University, 1400 Townsend Dr. Houghton, Michigan 49931-1295. e-mail: froese@mtu.edu

${ }^{1}$ Corresponding author (e-mail: caf@nibio.no). 


\begin{abstract}
We present a revised estimator for the sampling error of local competition variables that builds on the conceptual framework given by Stage \& Wykoff (Stage, A. R., and W. R. Wykoff. 1998. Adapting distance-independent forest growth models to represent spatial variability: effects of sampling design on model coefficients.

Forest Science 44(2): 224-238). Accurate estimation of the sampling error of local competition variables is a requisite for most approaches that correct the effects of measurement error in model fitting and application. Our revision addresses the bias inherent in Stage \& Wykoff's estimator due to the overlapping of random samples that are constrained to include a subject tree. We also argue that the adjustment Stage \& Wykoff used to account for the absence of treeless plots (zero-truncation) is unnecessary. We illustrate the performance of the new estimator and that of Stage \& Wykoff through simulation. For a hypothetical Poisson forest (800 trees/ha and mean dbh $9.8 \mathrm{~cm}$ ) bias is negligible for the new estimator, and variance is reduced by $92 \%$. Key words: measurement error, competition index, point basal area, PBA, structural based prediction.
\end{abstract}


17

18

19

\section{Introduction}

Competition among neighboring trees for limited resources plays an essential role in forest stand dynamics. Because competition affects each of the basic components of most tree growth models (Begon et al., 1996), it is broadly included in forest growth and yield simulation systems (Hann and Zumrawi, 1991). Competition may be described based on underlying mechanisms. Where trees share a pool of resources, competition may be "diffuse", and the effect of such competition "symmetric", because it affects all trees equally or in proportion to their size (Nilsson, 1994). Alternatively, competition may be "sharp", arising from interference (García, 1992), and the effect described as "asymmetric" because large trees affect the growth of small trees more than they are affected (Nilsson, 1994). Indices or metrics of competition may be used in growth models to represent these mechanisms, and implicit if not explicit in these indices is the definition of the competition neighborhood (Ledermann and Stage, 2001).

Growth and yield models commonly use indices of competition at two spatial scales: at the whole stand level, as an average, or at the local level. Competition metrics, regardless of their scale, are, like most measured variables, subject to measurement error. Stage and Ledermann (2008) provide a concise discussion of the sources of error intrinsic to competition variables. For example, error can arise from a discrepancy between the zone of influence defined in the variable and the actual relationship between a subject tree and its competitors, both diffuse and sharp. Both stand and local level metrics are subject to error arising from the variability inherent to point estimation that originates when competition metrics are estimated through sampling (Stage and Wykoff, 1998).

When competition variables are used as predictors in regression models, the potential effects of measurement error are significant. Under the classical measurement error model, both regression coefficients and model predictions are biased (Fuller, 1987; Canavan and Hann, 2004). The magnitude of the bias is a function of plot size and configuration, and this clearly complicates interpretation of regression parameters based on magnitude, within and among unrelated studies. In addition, bias in model predictions can arise through difference in the configuration of plots used to calibrate a model and plots used to initiate it, or when tree sizes change, trees are removed through management, or through estimation of mortality across 
46 the course of a simulation (Stage and Ledermann, 2008). The recognition of these critical ${ }_{47}$ latent risks has motivated sustained attention in forestry literature (Jaakkola, 1967; Hann and

48 Zumrawi, 1991; Stage and Wykoff, 1998; Lappi, 2005). Most of the approaches to correct for

49 measurement error assume some knowledge of the measurement error variance (Fuller, 1987;

${ }_{50}$ Carroll et al., 2006). The correct estimation of this variance is one of the most critical steps

51 in all of the most widely used approaches for correcting of measurement error (Fuller, 1987;

52 Carroll et al., 2006).

53 Estimates of the error variance can be obtained from internal replicates; that is, when 54 several measurements of the competition variable are available one can estimate the variance 55 with the usual sample-based estimator. This approach is straightforward for stand level metrics 56 like stand basal area. Unfortunately, standard forest inventories do not include replicates of 57 local competition variables, like plot basal area. Other options when internal replicates are not 58 available include estimating the variance from external studies or deriving it from the data 59 available in the inventory. Because the variance changes with the spatial structure, diameter 60 distribution and plot size (Stage and Wykoff, 1998) the former option is not feasible in practice.

61 This latter approach involves assumptions about the spatial pattern of tree locations, and 62 about the diameter correlation among neighboring trees. This last approach was selected by 63 Stage and Wykoff (1998), hereafter "S\&W", to implement the only methodology developed 64 until now that corrects for measurement error in competition variables in both the calibration 65 and application phases of diameter increment / basal area models.

The S\&W approach was motivated by the possibility that many randomly-located plots could contain a single subject tree. Their estimate of measurement error variance aims, therefore, to estimate the variance of local competition over all possible locations of the plot constrained to contain the subject tree. We argue that the variance estimator Stage and Wykoff (1998) derived should account for the overlapping areas of these plots, and therefore the S\&W estimator overestimates the variance of local competition. We also argue that the approach used to account for the truncated nature of the variance for the subject tree is unnecessary.

In this paper we describe an estimator that accounts for the overlapping area. We illustrate the performance of the new estimator and the $\mathrm{S} \& \mathrm{~W}$ estimator through a simulated Poisson forest. Our objective is to present improvements to the $\mathrm{S} \& \mathrm{~W}$ estimator and not to address the 
$\operatorname{Var}(M)=\operatorname{Var}\left(\frac{m}{a}\right)=\frac{\operatorname{Var}(m)}{a^{2}}=\frac{\mu}{a^{2}}=\frac{(\tau a)}{a^{2}}=\frac{\tau}{a}$ can be estimated as: its variance is:

assumptions about tree locations or the spatial correlation of tree attributes used in S\&W to derive the estimator for the competition variance. We begin with a description of the $\mathrm{S} \& \mathrm{~W}$ approach to the estimation of the local competition variance, followed by a rationale for and description of the proposed improved variance estimator. The last part of this paper presents a simulation that illustrate the performance of both the old and new variance estimators.

2. Stage and Wykoff (1998) approach to the estimation of the local competition variance

In this section we outline $\mathrm{S} \& \mathrm{~W}$ approach to estimate local competition variance. We follow S\&W notation. Population variances are indicated as $\operatorname{Var}($.$) , and variance for sample estimates$ as $\operatorname{var}($.$) . S\&W's approach is based on two main assumptions: (1) that trees are located follow-$ ing a random (Poisson) pattern and (2) that the diameter of each tree is independent of the locations of the remaining trees. Based on these assumptions the sampling variance of point basal area (PBA) in the neighborhood of the tree is estimated as follows.

In a Poisson forest, the variance of the number of trees per plot $(\mathrm{m}), \operatorname{Var}(m)$, can be estimated as the mean tree count per plot $(\mu)$, that is, $\operatorname{Var}(m)=\mu$, and $\mu=\tau \cdot a$, where $\tau$ is the mean number of trees per unit area, and $a$ is the area of the plot (e.g., ha).

Changing from statistics for the plot to statistics per unit area $M=m / a$, and $\operatorname{Var}(M)$

To derive the sampling distribution of basal area in a Poisson forest, divide the population into diameter classes indexed $i$ and sampled with plots of area $a_{i}$. Each class is itselfa Poisson subpopulation because of the way the Poisson forest is generated. Define $B A_{i}$ as the basal area in the $i^{\text {th }}$ diameter class with a midclass diameter of $d$, and variance $\operatorname{Var}\left(B A_{i}\right)$. The basal area per unit area is the total over the diameter classes, that is, $B A_{i}=c d_{i}^{2} m_{i} / a_{i}$, and 
$\operatorname{Var}\left(B A_{i}\right)=\frac{c^{2} d_{i}^{4} \operatorname{Var}\left(m_{i}\right)}{a_{i}^{2}}=\frac{c^{2} d_{i}^{4} \mu_{i}}{a_{i}^{2}}=\frac{c^{2} d_{i}^{4} \tau_{i}}{a_{i}}$

$\operatorname{var}_{i}(P B A)=c^{2}\left(\sum_{j=1}^{r} \frac{M_{j} d_{j}^{4}}{a_{j}}\right)-\frac{d_{t}^{4}}{a_{t}}$ is: $\mathrm{PBA}$ variance. The suggested $\mathrm{SF}$ is:
$\operatorname{Var}(P B A)=\sum_{i} \operatorname{Var}\left(B A_{i}\right)=c^{2} \sum_{i} \frac{d_{i}^{4} \tau_{i}}{a_{i}}$

Since any sample of the local competition around a particular tree includes that particular tree, the distribution of the diameter class of the subject tree is not a true Poisson distribution but it is truncated. S\&W accounted for this by subtracting $d_{t}^{4} / a_{t}$ from the equation. Therefore, the adjusted estimate of PBA sampling variance, vPBA, in the neighborhood of the $t$ th tree

where $r$ is the number of tree records left in the inventory of the sample point.

Stage and Wykoff claimed that samples with higher than average counts per point were more frequently overestimating the local mean and variance, and that samples with lower than average counts per point were more frequently underestimating the true mean and variance. To overcome this problem, they added a shrinkage factor (SF) which is a multiplier to the

$S F=\frac{P V \cdot S B A+(1-P V) P B A}{P B A}$ 
Where PV is a constant taking a value between 0 and 1 . S\&W found a value for PV empirically, based on the criterion of minimizing the error of the estimate of the resulting model. The selected PV was 1 , so $\mathrm{SF}=\mathrm{SBA} / \mathrm{PBA}$. The main effect of the shrinkage factor is to shrink the estimated variance from the single sample towards a central value for the stand (Stage and Wykoff, 1998).

\section{An improved variance estimator}

We argue that the reason behind the discrepancies between observed and estimated variance is that the possible sampling points around a tree have overlapping areas, and this is incongruent with some of the properties of the Poisson distribution.

One of the properties of a Poisson distribution is the memorylessness property (Cox and Isham, 1980). It is derived from this property that the number of events (trees) in an area is independent from the number of events (trees) in another area that is disjoint from the first area. However, if two areas are not disjoint, but are overlapping, the number of trees in one area is not independent of the number of trees in the other area (Figure 1). Since all possible samples including a tree must have an overlapping area that includes the subject tree, the plots are not disjoint, and thus, the number of trees in each plot is not independent of each other. In a Poisson forest with parameter $\lambda=200$ trees/ha, the expected number of trees in any sample plot, disjoint or not, of size 1 ha is 200 trees. However, the variance in the number of trees is $\lambda$ when the plots are disjoint, and less than $\lambda$ if the plots have an overlapping area. If we sample in a Poisson forest with parameter $\lambda$ in such a way that the expected value of the overlapping area of the plots of size $A_{p}$ and radius $r$ is $A_{O}$, the expected number of trees per plot would still be $\lambda \cdot A_{p}$ but the variance would be $\lambda \cdot\left(A_{p}-A_{O}\right)$, because the overlapping area does not contribute to the variance. Since the plot size, $A_{p}$, is known and $\lambda$ can be easily estimated, we focus on estimating $A_{O}$.

The expected overlapping area, $E\left(A_{O}\right)$, of two circles that always contain a fixed point can be defined in terms of the position, distances $\left(x_{1}, x_{2}\right)$ and angles $\left(\theta_{1}, \theta_{2}\right)$, of the centers of the two circles with respect to the fixed point as:

○2015 NRC Canada 


$$
E\left(A_{O}\right)=\int_{0}^{r} \int_{0}^{2 \pi} \int_{0}^{r} \int_{0}^{2 \pi} p\left(x_{1}, \theta_{1}, x_{2}, \theta_{2}\right) A\left(x_{1}, \theta_{1}, x_{2}, \theta_{2}\right) d x_{1} d \theta_{1} d x_{2} d \theta_{2}
$$

$p\left(x_{1}, \theta_{1}, x_{2}, \theta_{2}\right)=\frac{2 \pi x_{1}}{\pi r^{2}} \cdot \frac{2 \pi x_{2}}{\pi r^{2}} \cdot \frac{1}{2 \pi} \frac{1}{2 \pi}$

and $A\left(x_{1}, \theta_{1}, x_{2}, \theta_{2}\right)$ is the overlapping area of two circles with centers at distance $x_{1}$ and $x_{2}$ and angle $\theta_{1}$ and $\theta_{2}$ of the fixed point (Figure 2).

To calculate the overlapping area of two intersecting circles of radius $r$ and centers $a$ and $b$ (Figure 2), the angles $c a d$ and $c b d$, where $c$ and $d$ are the two points of intersection, are calculated using the law of cosines. To calculate the angle cad the angle cab, which is half of cad, is first calculated.

According to the law of cosines $r^{2}=r^{2}+x^{2}-2 r \cdot x \cdot \cos (c b a)$, so $\cos (c b a)=x / 2 r$, and

$c b d=2 c b a=2 \arccos (x / 2 r)$

Then, the segment of each of the circles cut off by the chord $c d$ is calculated by taking the area of the sector of the circle $b c d$ and subtracting the area of triangle $b c d$. Similarly, the area of the sector acd can be found and the area of triangle acd subtracted. That is,

Area $=A\left(x_{1}, \theta_{1}, x_{2}, \theta_{2}\right)=2\left[\frac{1}{2} c b d \cdot r^{2}-\frac{1}{2} r^{2} \cdot \sin (c b d)\right]=r^{2}(c b d-\sin (c b d))$

and substituting 7 in 8 we obtain:

$$
A\left(x_{1}, \theta_{1}, x_{2}, \theta_{2}\right)=r^{2}\left[2 \arccos \left(\frac{x}{2 r}\right)-\sin \left(2 \arccos \left(\frac{x}{2 r}\right)\right)\right]
$$


The distance between the centers of the circles, $x$, can be redefined in terms of $x_{1}, \theta_{1}, x_{2}, \theta_{2}$ from the coordinates for the centers as:

$$
\begin{gathered}
x=\sqrt{\left[a_{x}-b_{x}\right]^{2}+\left[a_{y}-b_{y}\right]^{2}}= \\
\sqrt{\left[\cos \left(\theta_{1}\right) x_{1}-\cos \left(\theta_{2}\right) x_{2}\right]^{2}+\left[\sin \left(\theta_{1}\right) x_{1}-\sin \left(\theta_{2}\right) x_{2}\right]^{2}}
\end{gathered}
$$

Integrating $E\left(A_{O}\right)$ in [5] results in $0.4596199 \pi r^{2}$. That is, the expected overlapping area is about $46 \%$ of the area of the circle, and only $54 \%$ of the area of the plot contributes to the variance. The new estimate $(n e)$ of the vPBA would then be:

$\operatorname{var}_{i}(P B A)=(1-0.4596199) c^{2}\left(\sum_{j=1}^{r} \frac{M_{j} d_{j}^{4}}{a_{j}}\right)$

The new estimate accounts for the overlapping area of the plots including the subject tree. However, we do not include the deduction for the subject tree proposed by Stage and Wykoff (1998). Since any sample of the local competition around a particular tree includes that particular tree, the distribution of the diameter class of the subject tree is not a "pure Poisson" but rather is zero-truncated Poisson. While the variance of a sum of Poisson variables is the sum of the individual Poisson variances, the variance of the sum of Poisson and a zerotruncated Poisson is not, and some adjustment is required. Intuitively, the certainty that a plot contains at least the subject tree should reduce the sampling error. To address this, Stage and Wykoff (1998) defined the diameter class of the subject tree as "vanishingly narrow", such that the subject tree would not contribute to the variance; in their formula they subtracted $d_{t}^{4} / a_{t}$ from the equation for $\operatorname{var}_{i}(P B A)$ [4]. However, for a zero-truncated Poisson, as the mean number of trees per plot increases, the effect of the zero truncation has a diminishing effect on the variance. In other words the variance of a truncated Poisson approaches the variance of a "pure Poisson" as the number of trees per plot increases, and thus the fixed correction proposed by Stage and Wykoff (1998) is not appropriate. Unfortunately, there seems to be no way to address this problem in a closed form, but for reasons we argue later the bias due to ignoring the zero-truncation of the class containing the subject tree should be minimal in most 
175

applications.

\section{An illustration of the behaviour of the estimators}

A straightforward way to test if the suggested variance estimator is an improvement over the S\&W variance estimator for Poisson forests is through simulation. We simulate spatiallyexplicit Poisson forest stands, simulate the measurement error variance, and finally compare the variance to the proposed estimator and the S\&W estimator with and without the shrinkage factor.

Stands were simulated using spatial point processes (Stoyan and Penttinen, 2000). A thorough introduction to point processes can be found in Diggle (2003), and for a more mathematical presentation see Stoyan et al. (1995). To generate the stands we used a spatial point process called homogeneous Poisson process. The homogeneous Poisson process requires only one parameter to be specified; the value we used to simulate the stands is $\lambda=0.08$ ( 800 trees/ha). 100 stands were generated using the same value for $\lambda$. Because point processes are stochastic, each realization differs from the rest of the realizations of the stand in tree location, total number of trees and other tree and stand characteristics. All created stands were square with dimension $150 \times 150 \mathrm{~m}$ (i.e., 2.25 ha).

We assigned diameters from the truncated Weibull distribution. The Weibull has been commonly used to describe diameter distributions of forest stands (Cao and Coble, 2014). Truncation is necessary because most inventories measure only trees above a minimum diameter, which is usually the smallest tree of merchantable size. Equations for the truncated Weibull function are given by Stiff (1979):

$f_{T}=\frac{c}{b}\left(\frac{x}{b}\right)^{c-1} e^{\left(\frac{T^{c}-x^{c}}{b^{c}}\right)}$

$F_{T}(x)=1-e\left(\frac{T}{b}\right)^{c} e^{-\left(\frac{x}{b}\right)^{c}}$ 
$x_{T}=b\left[\left(\frac{T}{b}\right)^{c}-\ln \left(1-F_{T}(x)\right)\right]^{1 / c}$

where $\mathrm{x}$ indicates $\mathrm{dbh}, \mathrm{f}(\mathrm{x})$ the probability density function, and $\mathrm{F}(\mathrm{x})$ the cumulative distribution function. We used $\mathrm{T}=5 \mathrm{~cm}, \mathrm{c}=0.87$, and $\mathrm{b}=15$.

\subsection{Sampling the stands}

The overall approach has two main parts. In the first, plot sampling is simulated on the 100 spatially-explicit stands. For each tree included in each sampling plot the measurement error variance is quantified and compared to alternative variance estimators.

For the first part of the approach four variances are calculated for each tree included in every plot:

1. true- the true PBA variance. Estimated by Monte Carlo simulation, by placing 100 random plots around each tree and calculating the PBA for each of these plots. It is assumed that the variance of these 100 replicates is a good approximation to the real variance of the PBA around the subject tree,

2. $S W$ - the PBA variance following Stage and Wykoff's methodology without the shrinkage factor,

3. $S W-S$ - the PBA variance following Stage and Wykoff's methodology with the shrinkage factor,

4. ne- new estimator

Four sampling plots were placed in each stand. The sampling plots were selected in such way that their areas did not intersect with each other and that their centers were at least twice the plot radius from the edge. Around each tree falling inside any of these four plots 100 new randomly placed plots were located. The variance of the PBA of these resampling plots was used to calculate true for each tree. These plots were of similar size and shape as the original sampling plots and were located in such a way that all 100 resampling plots included the subject tree. 

variance estimates $(S W, S W-S$ and $n e)$ and true. For example, in the case of $S W$, the RMSE was calculated as:

$R M S E=\sqrt{\frac{\sum_{i=1}^{n}\left(\text { true }_{i}-S W_{i}\right)^{2}}{n}}$

Data analysis, simulations and graphics were performed in the $\mathrm{R}$ environment ( $\mathrm{R}$ Development Core Team, 2012) and using the 'spatstat' package (Baddeley and Turner, 2005).

\subsection{Comparison of the variance estimators}

Both $S W$ and $S W-S$ substantially overestimate true variance (Figure 3). The RMSE for ne (1 463) is more than ten times smaller than the RMSE for $S W$ (21 232), or $S W-S$ (17 605). The SF of S\&W does slightly improve the estimates of vPBA, but $S W-S$ still overestimates vPBA. $S W$ and $S W-S$ are not only biased but have also a much wider range of values than true or ne with values nearly twice the largest values of true or ne.

The new estimator is substantially less biased than the S\&W estimator. The slight overestimation of the variance originates from not addressing the zero-truncation of the distribution of the diameter class represented by the subject tree. We expect this bias to be higher, relative to the vPBA values, at stands with low densities and smaller at stands with higher densities. In the illustration presented here, for stands with densities of 800 trees/ha, the bias is small compared to the values of vPBA $(7 \%)$.

\section{Discussion}

The effective use of techniques to account for the effect of measurement error of competition variables in empirical growth and yield modelling depends on correct specification of the error variance. The estimate derived by Stage and Wykoff (1998) for vPBA provided a useful initial framework for the study of measurement error in local competition variables. However, our simulation study shows that their approach produces a substantial overestimate of $\mathrm{vPBA}$, subject to assumptions about spatial randomness and independence of tree attributes. S\&W's 
addition of a shrinkage factor only marginally reduces this bias. In contrast, the new estimator we present, which accounts for the expected area of overlap in random samples constrained to include a subject tree, is nearly unbiased, and also has the desirable property of much reduced variance. We draw from the same conceptual framework as $\mathrm{S} \& \mathrm{~W}$, assuming a Poisson forest and dividing the plot into diameter classes, and the new estimator is simpler and computationally less intensive to calculate. Even if the assumptions about spatial randomness and independence do not hold, the issue of overlapping area persists and will need to be addressed. Our demonstration used circular fixed-area plots, but the estimator is equally applicable for Bitterlich plots, because the expected area of overlap is a fixed proportion of the plot size, and though plot size is a function of diameter, each diameter class is treated separately in the calculation of the measurement error.

Our new estimator, like the original proposed by $\mathrm{S} \& \mathrm{~W}$, depends on the assumption of a Poisson forest, in which both tree spatial location and essential attributes (i.e., dbh) are uncorrelated. This assumption is obviously unlikely to be strictly met in practice. Numerous studies of spatial patterns have revealed both attraction and repulsion in natural and managed stands at the scale of typical inventory plots, the precise domain of which is a function of initial condition, microsite, thinning, age, and the accumulated and dynamic effects of stand development (Reed and Burkhart, 1985; García, 1992; Reich and Arvanitis, 1992). When trees or attributes are clustered the Poisson assumption will result in underestimation of the sampling error, and in regular stands (such as plantations) result in overestimation. The asymmetric effect of "sharp" competition is likely antagonistic to the positive correlation induced by microsite, and the resulting temporal interactions are complex (García, 1992). Hence, and in the absence of compelling alternatives, Stage and Wykoff (1998) concluded that "the assumption of independent Poisson subpopulations seems a likely first approximation". We echo S\&W's recommendation that refinements will benefit from explicit study of mapped stands. In any case, the suitability of the Poisson assumption in any specific case does not diminish the utility of the improvements to the $\mathrm{S} \& \mathrm{~W}$ estimator we present here, which was our principal objective.

There are several reasons why we should seek an improved model for measurement error variance of local competition variables, like vPBA. One objective of the use of measurement error methods is to correct for the attenuation of regression coefficients (Fuller, 1987; Carroll 
et al., 2006); attenuation clearly complicates interpretation of the magnitude of coefficients within and across modelling contexts. This is especially important when the effect of measurement error is a complex function of stand history, inventory design, and management. The original S\&W estimate of vPBA overestimates the measurement error, which in application will lead to an overcorrection, and since the overestimate is higher in plots with higher PBA this would lead to overestimation of the effect of competition. Further, when measurement error methods are used in multiple regression models the relative weight attached to alternative competition variables can change due to the correction of the attenuation in variables measured with error. For example, Stage and Wykoff (1998) showed in their example using an individual-tree basal area increment model that correction for measurement error in PBA caused a shift in emphasis away from crown ratio (CR) and towards PBA. In the absence of disturbance, $\mathrm{CR}$ at least partially reflects the competitive status of the tree, but is included so that models can generate reasonable predictions when disturbance such as thinning is simulated (Wykoff, 1990). The shift in emphasis implies that the model corrected for measurement error would respond differently to disturbance. Thus, correct specification of measurement error may also be essential to effectively represent tree and stand dynamics. Assumptions about spatial pattern and the correlation of tree attributes will also affect the variance model, and though study of these aspects are outside of the scope of our study they are clearly important for the same reasons and should be investigated in future work.

The adjustment that S\&W proposed for zero-truncation in the diameter class that contains the subject tree has the effect of reducing bias, but largely not as intended. It is straightforward to show that the variance of a zero-truncated Poisson rapidly approaches the variance of a "pure Poisson" as the expected count increases. Thus, the reduction in variance due to zerotruncation should be a function of the expected value, not a constant as proposed by S\&W. We believe that the small bias that remains in our new estimator is due to ignoring the zerotruncation, especially in plots with few trees. However, we expect that the bias will be small in most conceivable applications. The greatest bias will occur with small plots or in sparse stands, because in both of these cases the expected number of trees per plot will be low. In practice, the size or configuration of forest inventory plots is usually adapted to stand conditions, such that a minimum number of trees are included at each sample location (Avery and Burkhart, 
2002; Gregoire and Valentine, 2008); thus, the presence of plots with very low counts should be uncommon, and if anticipated, the analyst can compensate through altering the sampling design. Furthermore, since basal area is a squared power of diameter, if stands are sparse because trees are small, then we expect both PBA and vPBA will also be small, and the bias from ignoring zero-truncation will be of less practical significance. In the case where local density (e.g., trees per unit area) is used as a local competition variable, then trees are treated equally and the expected measurement error variance can be calculated using a zero-truncated Poisson directly, still following the logic of assuming the parameter of the now zero-truncated Poisson forest varies spatially and the tree counts at each plot are estimates of the parameter of the local population.

The new estimator proposed here can be applied to any growth and yield model that uses local competition variables such as point basal area or number of trees. Implementing the new estimator should enhance the already proven abilities of measurement error techniques to increase the predictive ability of the models.

\section{Literature cited}

References

Avery, T.E., Burkhart, H.E., 2002. Forest measurements. 5th ed. McGraw-Hill, New York, NY.

Baddeley, A., Turner, R., 2005. Spatstat: an r package for analyzing spatial point patterns. Journal of Statistical Software 12, 1-42.

Begon, M., Harper, J.L., Townsend, C.R., 1996. Ecology: individuals, populations, and communities. Blackwell Science, Oxford,UK.

Canavan, S.J., Hann, D.W., 2004. The two-stage method for measurement error characterization. For. Sci. 50, 743.

Cao, Q.V., Coble, D.W., 2014. Deriving a diameter distribution form stand table data. For. Sci. 60. 
330

Carroll, R.J., Ruppert, D., Stefanski, L.A., Crainiceanu, C.M., 2006. Measurement error in nonlinear models. Chapman and Hall/CRC Press, Boca Raton, FL.

Cox, D.R., Isham, V., 1980. Point processes. volume 12. Chapman \& Hall/CRC, London.

Diggle, P.J., 2003. Statistical analysis of spatial point patterns. Oxford University Press, New York. second edition edition.

Fuller, W.A., 1987. Measurement error models. John Wiley and Sons, New York, NY.

García, O., 1992. What is a diameter distribution, in: Proceedings of the Symposium on Integrated Forest Management Information Systems, Tsukuba, Japan. pp. 11-29.

Gregoire, T.G., Valentine, H.T., 2008. Sampling strategies for natural resources and the environment. Chapman and Hall/CRC Press, Boca Raton, FL.

Hann, D.W., Zumrawi, A.A., 1991. Growth model predictions as affected by alternative sampling-unit designs. For. Sci. 37, 1641-1655.

Jaakkola, S., 1967. On the use of variable size plots for increment research, in: Proceedings of XIV. IUFRO-Congress Sect. 25., DVFFA, Munich. p. 371-378.

Johnson, N.L., Kotz, S., 1969. Distributions in statistics: discrete distributions. volume 1. Houghton Mifflin, Boston.

Lappi, J., 2005. Plot size related measurement error bias in tree growth models. Can. J. For. Res. 35, 1031-1040.

Ledermann, T., Stage, A.R., 2001. Effects of competition spacing in individual-tree indices of competition. Can. J. For. Res. 31, 2143-2150.

Nilsson, U., 1994. Development of growth and stand structure in Picea abies stands planted at different initial densities. Scandinavian Journal of Forest Research 9, 135-142.

R Development Core Team, 2012. R: A language and environment for statistical computing. Reed, D.D., Burkhart, H.E., 1985. Spatial autocorrelation of individual tree characteristics in loblolly pine stands. For. Sci. 31, 575-594.

이리 NRC Canada 
355

Reich, R.M., Arvanitis, L.G., 1992. Sampling Unit, Spatial Distribution of Trees, and Precision. North. J. Appl. For. 9, 3-6.

Stage, A.R., Ledermann, T., 2008. Effects of competitor spacing in a new class of individualtree indices of competition: semi-distance-independent indices computed for bitterlich versus fixed-area plots. Can. J. For. Res. 38, 890-898.

Stage, A.R., Wykoff, W.R., 1998. Adapting distance-independent forest growth models to represent spatial variability: effects of sampling design on model coefficients. For. Sci. 44, $224-238$.

Stiff, C.T., 1979. Modeling the growth dynamics of natural mixed-species Appalachian hardwood stands. Dissertation. Virginia Polytechnic Institute and State University. Blacksburg, VA.

Stoyan, D., Kendall, W.S., Mecke, J., 1995. Stochastic geometry and its applications. volume 2nd ed. Wiley, Chichester.

Stoyan, D., Penttinen, A., 2000. Recent applications of point process methods in forestry statistics. Statistical Science 15, 61-78.

Wykoff, W.R., 1990. A basal increment model for individual conifers in the northern rocky mountains. For. Sci. 36, 1077-1104. 
Fig. 1. Any potential plot (grey areas) containing the subject tree (white cross) overlap.

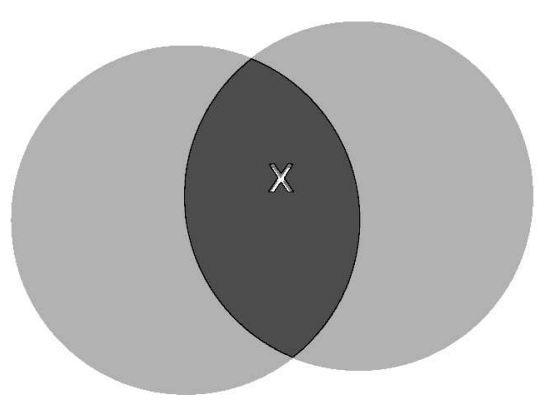


Fig. 2. The expected overlapping area of two circles of radius $r$ and centres $a$ and $b$, that always contain a fixed point, can be defined in terms of the angles $\theta_{1}$ and $\theta_{2}$ and the distances $x_{1}$ and $x_{2}$. The distance between the centres is $x$, and $c$ and $d$ are the intersection points of the circumference of the two circles.
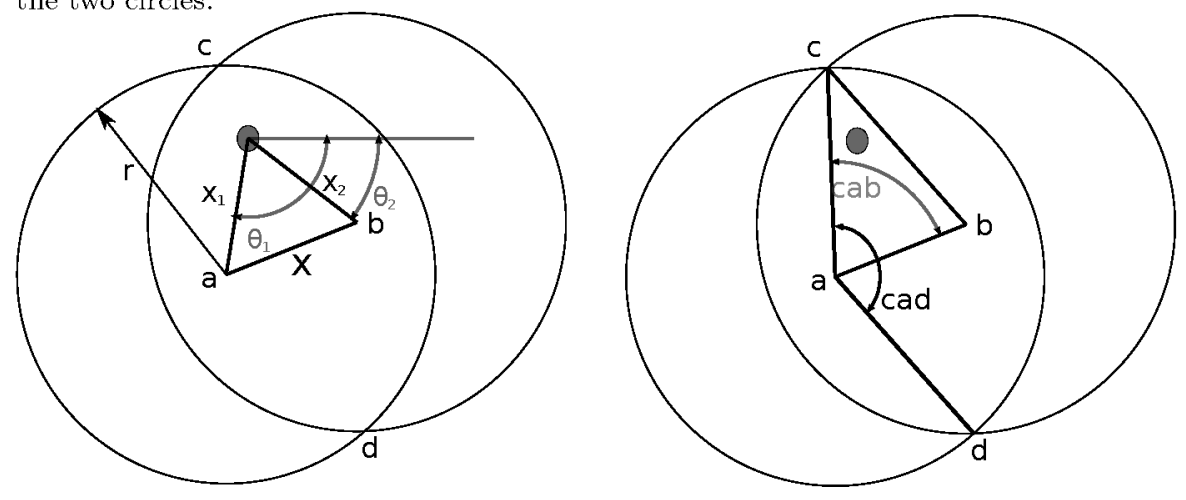
Fig. 3. Violin plots of the sampling variance, vPBA, estimated using Monte Carlo simulations (true), Stage and Wykoff's methodology with $(S W-S)$ and without the shrinkage factor $(S W)$, and the new estimator (ne). The solid circle shows the median, and the rectangle the two inner quartiles of the distribution of values. The outer envelope is a kernel density of the entire set.

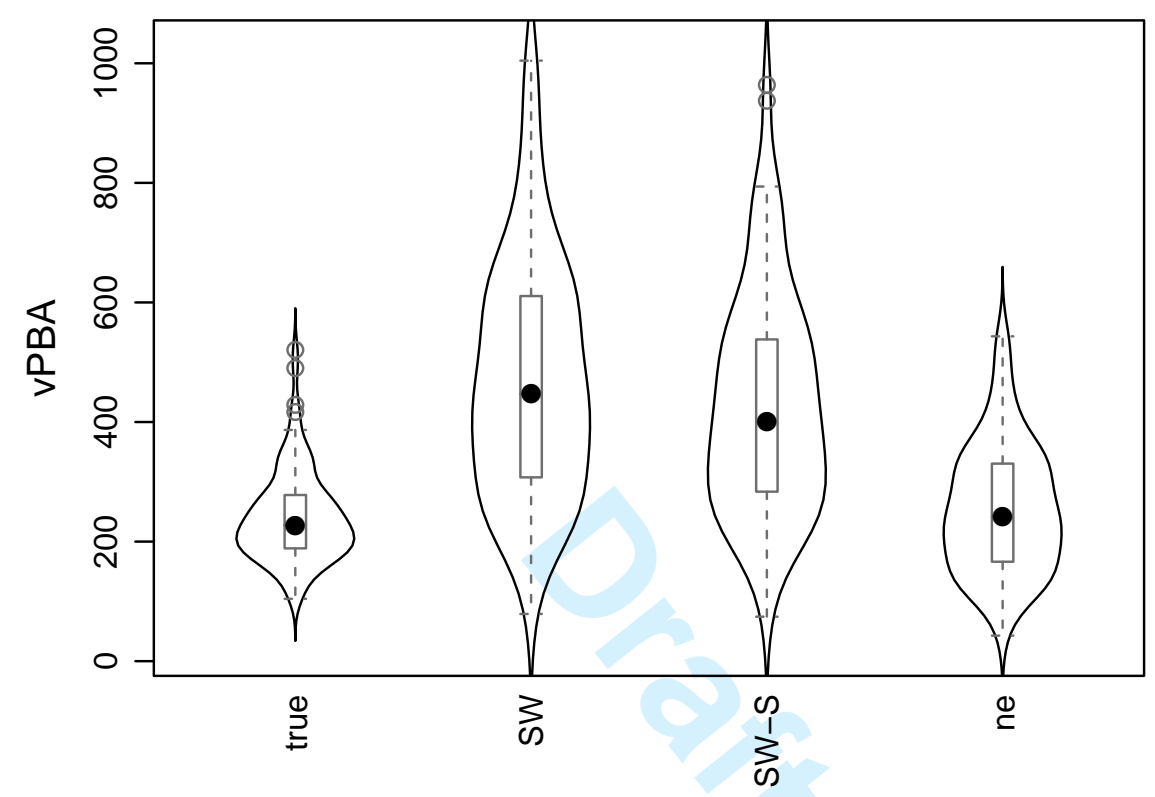

○2015 NRC Canada 